\title{
Almost Disturbance Decoupling for a Class of Fractional-Order Nonlinear Systems with Zero Dynamics
}

\author{
Xiaoping Liu $\mathbb{D},{ }^{1}$ Yajing Zhao $\mathbb{D},{ }^{2}$ Caiyun Wang $\mathbb{D},{ }^{3}$ Huanqing Wang $\mathbb{D},{ }^{2,4}$ \\ and Yucheng Zhou iD ${ }^{2}$ \\ ${ }^{1}$ Faculty of Engineering, Lakehead University, Thunder Bay, ON P7B 5E1, Canada \\ ${ }^{2}$ School of Information and Electrical Engineering, Shandong Jianzhu University, Jinan, China \\ ${ }^{3}$ School of Science, Shandong Jianzhu University, Jinan, China \\ ${ }^{4}$ The Department of Mathematics, Bohai University, Jinzhou 12100, China \\ Correspondence should be addressed to Xiaoping Liu; xliu2@lakeheadu.ca
}

Received 24 December 2019; Revised 19 February 2020; Accepted 28 February 2020; Published 14 April 2020

Academic Editor: Wenqin Wang

Copyright ( 2020 Xiaoping Liu et al. This is an open access article distributed under the Creative Commons Attribution License, which permits unrestricted use, distribution, and reproduction in any medium, provided the original work is properly cited.

The problem of almost disturbance decoupling is addressed for fractional-order nonlinear systems. A new definition for the norm is proposed to describe the effect of disturbances on the output tracking error for fractional-order systems. Based on the Lyapunov stability theory and the backstepping design method, a tracking controller is constructed to make the output tracking error converge to zero without external disturbances and to attenuate the effect of disturbances on the tracking error at zero initial conditions. In order to validate these theoretical results, a numerical example and two practical examples are given.

\section{Introduction}

Recently, there has been a continuous growth in the research of fractional-order systems (FOSs). Thanks to the great efforts contributed by researchers, a large number of research results have been published on FOSs, see [1, 2]. It has been proved that fractional-order calculus can model systems more concisely than integer-order calculus [3]. Therefore, FOSs have been applied in numerous areas.

The output tracking control problem is one of the most important research topics. It is worth mentioning that the output tracking control has been widely applied in engineering systems including industry and economics [4-6]. As for FOSs, few research outcomes are published. An output tracking controller for a class of fractional-order positive switched systems is developed via an observer-based controller method [7]. In [8], a novel adaptive iterative learning sliding mode controller is proposed to control FOSs. What is more, the output tracking controllers can also be developed with the help of backstepping technique for FOSs. For instance, Wang et al. addressed an adaptive fuzzy output tracking control law for a class of uncertain FOSs with backstepping technique [9]. An output feedback neural network tracking controller for fractional-order nonlinear systems is constructed with the backstepping method [10].

Over the past decades, backstepping has become a widely used technique for adaptive nonlinear control. The technique was comprehensively addressed [11], and the basic idea of the backstepping scheme is to design a controller recursively by using a set of virtual controllers. The integer-order backstepping technique has been widely applied in many fields $[12,13]$. An adaptive finite-time fuzzy funnel controller is presented for nonaffine nonlinear systems via backstepping technique [14]. An event-triggered adaptive tracking controller based on a new funnel function for uncertain nonlinear systems is proposed by using backstepping technique [15]. Few research outcomes applying backstepping technique to FOSs are published. In [16], synchronization of two fractional-order chaotic Coullet systems is investigated based on the adaptive backstepping. A novel adaptive control technique named adaptive fractional-order backstepping is proposed for fractional-order nonlinear systems (FONSs) with uncertain constant parameters [17]. In [18], Ding et al. extended the classical backstepping to fractional-order backstepping for stabilising FONSs. Then, the pseudo-state 
stabilization problem of commensurate FONSs is constructed via the fractional-order backstepping scheme [19]. Wei et al. proposed a new technique to construct an adaptive backstepping controller for noncommensurate FOSs with the triangular form [20], and Wei et al. originally presented an adaptive output feedback control law for fractional-order systems by using the backstepping method [21]. Owing to most of the results which construct Lyapunov functions limited to the use of the frequency distribution model or linear matrix inequality (LMI), and the fractional-order backstepping techniques are still to be further studied.

It is well known that disturbances exist widely in industrial processes. The issue of disturbance decoupling has drawn great attention. The exact disturbance decoupling problem is to design a feedback controller so that the disturbance does not affect the output of the closed-loop system, which can be solved by the state or output feedback under some strict conditions [22-24]. However, for many practical systems, the exact disturbance decoupling problem is not solvable because the strict conditions are not satisfied. Therefore, the almost disturbance decoupling (ADD) problem is proposed by Weiland and Willems [25], which is to design a feedback controller such that the closed-loop system is stable and the $L_{2}$ gain from the disturbance to the output is less than or equal to an arbitrary positive constant. Up to now, there have been many research outcomes for the ADD problem for both linear and nonlinear control systems. For example, the ADD problem for SISO nonlinear systems is proposed [26]. The ADD problem of MIMO nonlinear systems and its application to chemical processes are addressed in [27]. In [28], Lin et al. discussed the problem of ADD for nonlinear systems with unstable zero dynamics. Also, in [29], Lin et al. developed a global result about the ADD problem for nonlinear systems with unstable zero dynamics. In [30], the authors investigated the problem of nonlinear output feedback tracking with almost disturbance decoupling. What is more, the ADD problem for high-order nonlinear systems is addressed by [31]. Tracking and almost disturbance decoupling for nonlinear systems with uncertainties are solved by Chen et al. [32]. Chen et al. [33] developed a direct adaptive fuzzy almost disturbance decoupling control method for nonlinear MIMO systems with unknown nonlinearities. For more research results on the ADD problem, see [34-38]. However, the research studies mentioned above are conducted only for integerorder systems. To the best of our knowledge, there are no reports on the ADD problem for FOSs.

The active disturbance rejection control (ADRC) problem, which utilizes an estimation and cancellation method to attenuate the negative impact of internal unmodelled dynamics and external disturbances, has been paid extensive attention recently. The ADRC problem has been addressed for nonlinear systems [39-44].

Motivated by the aforementioned reviews, this article develops an adaptive backstepping control algorithm to solve the problem of ADD for a class of fractional-order nonlinear systems. To this end, the following two challenges have to be overcome:
(1) Aghababa [45] has proved that Lin et al. [46] mistakenly applied the integer-order integral to deal with the fractional-order differential, which causes that the proposed controller in [46] cannot guarantee the $H_{\infty}$ performance of the closed-loop system, which implies that the $L_{2}$-norm cannot be directly used to solve the ADD problem. To solve this problem, a new form of norm based on the definition of Caputo fractional integral is proposed.

(2) When the backstepping design method is used, the fractional-order derivative of the virtual controller is required, which is a function of state variables. Therefore, the fractional-order derivative of a composite function is necessary. Unlike the integerorder derivative, the fractional-order derivative of a composite function is so complicated that it cannot be used to the fractional-order derivative of the virtual controller. To avoid such a challenge, the nonlinear functions in all the subsystems but last one are assumed to be linear.

Compared with the existing results, the main contributions are listed as follows:

(1) The problem of ADD is first addressed for a class of fractional-order nonlinear systems with external disturbances and zero dynamics.

(2) A new form of the norm based on the definition of Caputo fractional integral is proposed in order to prove the $H_{\infty}$ tracking performance for FOSs.

(3) With the help of backstepping technique, a tracking feedback control law is constructed, which ensures that the effect of external disturbances on the output tracking error is attenuated. A new form of Lyapunov function is chosen without the use of the frequency distribution model or LMI.

The remaining parts are arranged as follows. In Section 2, several definitions and useful lemmas relevant to the fractional-order calculus are shown. Section 3 proposes the problem formulation and gives the aim of this paper. In Section 4, the main results of this paper are presented. Section 5 gives a numerical example and two practical examples to further support the theoretical results. In the end, the conclusions are summarized in Section 6.

\section{Preliminaries}

\subsection{Fractional Calculus}

Definition 1 (Caputo fractional derivative [3]). Let $f(t)$ have continuous derivatives up to order $n-1$, i.e., $f^{(n-1)}(t)$ is a real continuously differentiable function. The Caputo fractional-order derivative of order $\alpha$ of $f(t)$ is defined by

$$
t_{0} D_{t}^{\alpha} f(t)=\frac{1}{\Gamma(n-\alpha)} \int_{t_{0}}^{t} f^{(n)}(\sigma)(t-\sigma)^{n-\alpha-1} \mathrm{~d} \sigma,
$$

where $0 \leq n-1<\alpha \leq n, n \in \mathbb{N}$, and $\Gamma(\cdot)$ is the gamma function. 
For simplicity in notations, ${ }_{t_{0}} D_{t}^{\alpha}$ is denoted as $D^{\alpha}$ for $t_{0}=0$.

Definition 2 (Caputo fractional integral [3]). Assume that $f(t)$ is an integrable function. Its Caputo fractional integral of order $\alpha$ is defined by

$$
{ }_{t_{0}} D_{t}^{-\alpha} f(t)=\frac{1}{\Gamma(\alpha)} \int_{t_{0}}^{t} f(\sigma)(t-\sigma)^{\alpha-1} \mathrm{~d} \sigma,
$$

where $\alpha>0$ and $t_{0}$ is the lower terminal.

For convenience in notations, $t_{0} D_{t}^{-\alpha}$ is denoted by $D^{-\alpha}$ for $t_{0}=0$.

Properties of the Caputo fractional calculus, which are used in the paper, are shown as Lemmas 1 and 2 .

Lemma 1 (an inequality of the Caputo fractional derivative [2]). For a differentiable function $x(t) \in \mathbb{R}^{n}$, a positivedefinite matrix $P \in \mathbb{R}^{n \times n}$, and $0<\alpha<1$, the inequality

$$
\frac{1}{2} D^{\alpha}\left(x^{T}(t) P x(t)\right) \leq x^{T}(t) P D^{\alpha} x(t),
$$

is true for any $t \geq 0$.

Lemma 2 (see [47]). Assume that $0 \leq n-1<\alpha \leq n$ and $f(t)$ is differentiable up to order $n-1$, that is, $f^{(n-1)}(t)$ is a differentiable function. Then,

$$
{ }_{t_{0}} D_{t}^{-\alpha}\left({ }_{t_{0}} D_{t}^{\alpha} f(t)\right)=f(t)-\sum_{k=0}^{n-1} \frac{f^{(k)}\left(t_{0}\right)}{k !}\left(t-t_{0}\right)^{k} .
$$

In particular, for a continuous function $f(t)$ and $0<\alpha \leq 1$, the following holds:

$$
{ }_{t_{0}} D_{t}^{-\alpha}\left(t_{0} D_{t}^{\alpha} f(t)\right)=f(t)-f\left(t_{0}\right) .
$$

2.2. Stability of Fractional-Order Systems. Consider the following FOS:

$$
{ }_{t_{0}} D_{t}^{\alpha} x(t)=f(x, t),
$$

where $t$ stands for the time and $0<\alpha \leq 1$.

Definition 3. A function $\beta:[0, t) \longrightarrow[0, \infty)$ is said to be a class- $\mathscr{K}$ function if it is continuous and strictly increasing with $\beta(0)=0$.

$\mathrm{Li}$ et al. [48] extended the Lyapunov direct method to FOSs, which is shown as Lemma 3.

Lemma 3 (see [48]). If there exist class- $\mathscr{K}$ functions $\beta_{i}(i=$ $1,2,3)$ and a function $V(x, t)$ so that

$$
\beta_{1}(\|x\|) \leq V(x, t) \leq \beta_{2}(\|x\|),
$$

and

$$
{ }_{t_{0}} D_{t}^{\alpha} V(x, t) \leq-\beta_{3}(\|x\|),
$$

with $0<\alpha<1$, then the equilibrium point $x=0$ of (6) is asymptotically stable.
Lemma 4 (Young's inequality). The inequality

$$
x^{T} y \leq \frac{\varepsilon^{p}}{p}\|x\|^{p}+\frac{1}{q \varepsilon^{q}}\|y\|^{q},
$$

holds for any $x, y \in \mathbb{R}^{r}, \varepsilon>0, p>1$, and $q>1$ with $(p-1)(q-1)=1$.

\section{Problem Formulation}

Consider a fractional-order strict-feedback nonlinear system with unknown disturbances and zero dynamics defined by

$\left\{\begin{array}{l}D^{\alpha} z(t)=f_{0}(z(t)), \\ D^{\alpha} x_{1}(t)=x_{2}(t)+f_{1}\left(\bar{x}_{1}(t)\right)+P_{1} \omega(t), \\ D^{\alpha} x_{2}(t)=x_{3}(t)+f_{2}\left(\bar{x}_{2}(t)\right)+P_{2} \omega(t), \\ \vdots \\ D^{\alpha} x_{n-1}(t)=x_{n}(t)+f_{n-1}\left(\bar{x}_{n-1}(t)\right)+P_{n-1} \omega(t), \\ D^{\alpha} x_{n}(t)=f(x(t))+g(x(t)) u(t)+f_{n}\left(\bar{x}_{n}(t)\right)+P_{n} \omega(t), \\ y(t)=x_{1}(t),\end{array}\right.$

where $0<\alpha<1, z(t)$ denotes the zero dynamics of (10), and $f_{0}(z(t))$ is a known nonlinear function with respect to $z(t)$. $x(t)=\left[x_{1}(t), \ldots, x_{n}(t)\right]^{T} \in \mathbb{R}^{n}$ and $\bar{x}_{i}(t)=\left[x_{1}(t), \ldots\right.$, $\left.x_{i}(t)\right]^{T} \in \mathbb{R}^{i}$ with $x_{i}(t)$ being the state of the system, while $y(t) \in \mathbb{R}$ and $u(t) \in \mathbb{R}$ are the system output and input, respectively. $\omega(t) \in \mathbb{R}^{r}$ represents the disturbance signal. $f_{i}\left(\bar{x}_{i}(t)\right)=a_{i} \bar{x}_{i}(t) \in \mathbb{R}(i=1, \ldots, n)$ with $a_{i}$ being a known vector. $f(\cdot)$ and $g(\cdot)$ are assumed to be nonlinear and known functions. $P_{i} \in \mathbb{R}^{1 \times r}$ is a known vector for $i=1, \ldots, n$. For convenience, denote $f(x(t)), g(x(t))$, $f_{0}(z(t)), f_{i}\left(\bar{x}_{i}(t)\right), \bar{x}_{i}(t), x_{i}(t)$, and $z(t)$ as $f, g, f_{0}, f_{i}, \bar{x}_{i}$, $x_{i}$, and $z$, respectively.

The aim is to construct a state feedback controller to drive the output $y(t)$ to track a desired reference signal $y_{r}(t)$, i.e., $\lim _{t \rightarrow \infty}\left(y(t)-y_{r}(t)\right)=0$ when $\omega(t)=0$ and to have an $H_{\infty}$ tracking performance for the zero initial state, i.e.,

$$
\left\|\left(y(t)-y_{r}(t)\right)\right\|_{\alpha} \leq \gamma\|\omega(t)\|_{\alpha},
$$

where $\|\cdot\|_{\alpha}$ is defined later and $\gamma>0$.

\section{Main Results}

4.1. The $L_{\alpha}$ Norm. In this section, a new form of norm named $L_{\alpha}$ norm based on the definition of Caputo fractional integral is presented.

Definition 4. The $L_{\alpha}$ norm of a function $\omega(t)$ is defined by

$$
\|\omega(t)\|_{\alpha}=\lim _{t \longrightarrow \infty} \sqrt{\int_{0}^{t} \omega^{T}(\sigma)(t-\sigma)^{\alpha-1} \omega(\sigma) \mathrm{d} \sigma},
$$

where $0<\alpha<1$.

This $L_{\alpha}$ norm satisfies three conditions of the norm, i.e., nonnegative (equation (13)), homogeneity (equation (14)), and triangle inequality (equation (15)). 
Lemma 5. The $L_{\alpha}$ norm satisfies the following properties:

(i) Nonnegative:

$$
\begin{aligned}
& \|\omega(t)\|_{\alpha} \geq 0 ; \\
& \|\omega(t)\|_{\alpha}=0, \quad \text { if and onl } y \text { if } \omega(t)=0 .
\end{aligned}
$$

(ii) Homogeneity:

$$
\|k \omega(t)\|_{\alpha}=|k|\|\omega(t)\|_{\alpha} .
$$

(iii) Triangle inequality:

$$
\left\|\omega_{1}(t)+\omega_{2}(t)\right\|_{\alpha} \leq\left\|\omega_{1}(t)\right\|_{\alpha}+\left\|\omega_{2}(t)\right\|_{\alpha} .
$$

Proof

(i) According to Definition (12), it can be easily verified that (13) is true.

(ii) Clearly,

$$
\begin{aligned}
\|k \omega(t)\|_{\alpha} & =\lim _{t \rightarrow \infty} \sqrt{\int_{0}^{t} k^{2} \omega^{T}(\sigma)(t-\sigma)^{\alpha-1} \omega(\sigma) \mathrm{d} \sigma} \\
& =|k|\|\omega(t)\|_{\alpha} .
\end{aligned}
$$

(iii) For convenience, denote $\omega(t)$ as $\omega$. It can be seen that the proof of condition (15) is equivalent to verify

$$
\left\|\omega_{1}+\omega_{2}\right\|_{\alpha}^{2} \leq\left(\left\|\omega_{1}\right\|_{\alpha}+\left\|\omega_{2}\right\|_{\alpha}\right)^{2}
$$

On the one hand,

$$
\begin{aligned}
\left\|\omega_{1}+\omega_{2}\right\|_{\alpha}^{2} & =\lim _{t \rightarrow \infty} \int_{0}^{t}\left(\omega_{1}+\omega_{2}\right)^{T}(t-\sigma)^{\alpha-1}\left(\omega_{1}+\omega_{2}\right) \mathrm{d} \sigma \\
& =\lim _{t \rightarrow \infty} \int_{0}^{t}\left(\omega_{1}^{T} \omega_{1}+2 \omega_{1}^{T} \omega_{2}+\omega_{2}^{T} \omega_{2}\right)(t-\sigma)^{\alpha-1} \mathrm{~d} \sigma \\
& =\left\|\omega_{1}\right\|_{\alpha}^{2}+\left\|\omega_{2}\right\|_{\alpha}^{2}+2 \lim _{t \rightarrow \infty} \int_{0}^{t} \omega_{1}^{T}(t-\sigma)^{\alpha-1} \omega_{2} \mathrm{~d} \sigma .
\end{aligned}
$$

On the other hand,

$$
\begin{aligned}
\left(\left\|\omega_{1}\right\|_{\alpha}+\left\|\omega_{2}\right\|_{\alpha}\right)^{2}= & \left\|\omega_{1}\right\|_{\alpha}^{2}+2\left\|\omega_{1}\right\|_{\alpha}\left\|\omega_{2}\right\|_{\alpha}+\left\|\omega_{2}\right\|_{\alpha}^{2} \\
= & \left\|\omega_{1}\right\|_{\alpha}^{2}+\left\|\omega_{2}\right\|_{\alpha}^{2} \\
& +2 \lim _{t \rightarrow \infty}\left[\sqrt{\left(\int_{0}^{t} \omega_{1}^{T}(t-\sigma)^{\alpha-1} \omega_{1} \mathrm{~d} \sigma\right)}\right] \\
& \cdot\left[\sqrt{\left(\int_{0}^{t} \omega_{2}^{T}(t-\sigma)^{\alpha-1} \omega_{1} \mathrm{~d} \sigma\right)}\right] .
\end{aligned}
$$

It is a simple exercise to show that the proof of (17) is equivalent to prove

$$
\begin{aligned}
& {\left[\int_{0}^{t} \omega_{1}^{T}(t-\sigma)^{\alpha-1} \omega_{2} \mathrm{~d} \sigma\right]^{2}} \\
& \leq\left[\int_{0}^{t} \omega_{1}^{T}(t-\sigma)^{\alpha-1} \omega_{1} d \sigma\right] \cdot\left[\int_{0}^{t} \omega_{2}^{T}(t-\sigma)^{\alpha-1} \omega_{2} \mathrm{~d} \sigma\right] .
\end{aligned}
$$

In order to verify (20), we construct a quadratic function of $u$ as follows:

$$
\begin{aligned}
\Phi(u)= & u^{2} \int_{0}^{t} \omega_{1}^{T}(\sigma)(t-\sigma)^{\alpha-1} \omega_{1}(\sigma) \mathrm{d} \sigma \\
& +2 u \int_{0}^{t} \omega_{1}^{T}(\sigma)(t-\sigma)^{\alpha-1} \omega_{2}(\sigma) \mathrm{d} \sigma \\
& +\int_{0}^{t} \omega_{2}^{T}(\sigma)(t-\sigma)^{\alpha-1} \omega_{2}(\sigma) \mathrm{d} \sigma .
\end{aligned}
$$

After a little manipulation, one can get the following result:

$$
\begin{aligned}
\Phi(u)= & \int_{0}^{t}\left[u^{2} \omega_{1}^{T}(\sigma)(t-\sigma)^{\alpha-1} \omega_{1}(\sigma)\right. \\
& +2 u \omega_{1}^{T}(\sigma)(t-\sigma)^{\alpha-1} \omega_{2}(\sigma) \\
& \left.+\omega_{2}^{T}(\sigma)(t-\sigma)^{\alpha-1} \omega_{2}(\sigma)\right] \mathrm{d} \sigma \\
= & \int_{0}^{t}(t-\sigma)^{(\alpha-1) / 2}\left[u \omega_{1}(\sigma)+\omega_{2}(\sigma)\right]^{T} \\
& \cdot\left[u \omega_{1}(\sigma)+\omega_{2}(\sigma)\right] \mathrm{d} \sigma \\
\geq & 0 .
\end{aligned}
$$

It is easily seen from (21) and (22) that the quadratic function intersects the $u$-axis at most one point, which implies that

$$
\begin{gathered}
{\left[2 \int_{0}^{t} \omega_{1}^{T}(\sigma)(t-\sigma)^{\alpha-1} \omega_{2}(\sigma) \mathrm{d} \sigma\right]^{2}} \\
-4\left[\int_{0}^{t} \omega_{1}^{T}(\sigma)(t-\sigma)^{\alpha-1} \omega_{1}(\sigma) \mathrm{d} \sigma\right] \\
\cdot\left[\int_{0}^{t} \omega_{2}^{T}(\sigma)(t-\sigma)^{\alpha-1} \omega_{2}(\sigma) \mathrm{d} \sigma\right] \leq 0 .
\end{gathered}
$$

which is equivalent to (20).

\subsection{Control Laws for $H_{\infty}$ Tracking Performance}

Assumption 1. For the zero dynamics $D^{\alpha} z=f_{0}(z)$ in system (10), there exists a positive-definite differentiable function $V_{0}$ so that its Caputo fractional derivative with order $0<\alpha<1$ satisfies the following inequality:

$$
D^{\alpha} V_{0}<0 \text {. }
$$


Assumption 2. The known nonlinear function $g(\cdot)$ satisfies the following inequality:

$$
|g(\cdot)|>0
$$

Assumption 3. The desired reference signal $y_{r}(t)$ and $\widetilde{D}_{i}$ are bounded and piecewise continuous, where $\widetilde{D}_{i}=\underbrace{D^{\alpha}\left(D^{\alpha}\left(D^{\alpha}\left(\cdots D^{\alpha}\right.\right.\right.}_{i}\left(y_{r}\right)))), i=0,1,2, \ldots, n$.

Define the control law by

$$
\begin{aligned}
u= & \frac{1}{g}\left[-f-k_{n} z_{n}-z_{n-1}-f_{n}+\sum_{s=1}^{n-1} \frac{\partial \alpha_{n-1}}{\partial x_{s}}\left(x_{s+1}+f_{s}\right)\right. \\
& \left.+\sum_{s=0}^{n-1} \frac{\partial \alpha_{n-1}}{\partial \widehat{D}_{s}} \widehat{D}_{s+1}-\frac{\widehat{P}_{n} \widehat{P}_{n}^{T}}{4 \gamma_{n}} z_{n}\right],
\end{aligned}
$$

and the virtual control laws by

$$
\left\{\begin{array}{l}
\alpha_{0}=y_{r}(t), \\
\alpha_{1}=-k_{1} z_{1}-f_{1}+D^{\alpha} \alpha_{0}-\frac{P_{1} P_{1}^{T}}{4 \gamma_{1}} z_{1}, \\
\ldots \\
\alpha_{i}=-k_{i} z_{i}-z_{i-1}-f_{i}+\sum_{s=1}^{i-1} \frac{\partial \alpha_{i-1}}{\partial x_{s}}\left(x_{s+1}+f_{s}\right) \\
\quad+\sum_{s=0}^{i-1} \frac{\partial \alpha_{i-1}}{\partial \widehat{D}_{s}} \widehat{D}_{s+1}-\frac{\widehat{P}_{i} \widehat{P}_{i}^{T}}{4 \gamma_{i}} z_{i}, \quad i=2, \ldots, n-1,
\end{array}\right.
$$

where

$$
\begin{gathered}
\left\{\begin{array}{l}
z_{1}=x_{1}-\alpha_{0}, \\
z_{i}=x_{i}-\alpha_{i-1}, \\
i=2,3, \ldots, n,
\end{array}\right. \\
\widehat{P}_{i}=P_{i}-\sum_{s=1}^{i-1} \frac{\partial \alpha_{i-1}}{\partial x_{s}} P_{s}, \quad i=2,3, \ldots, n,
\end{gathered}
$$

and

$$
\widehat{D}_{i}=\underbrace{D^{\alpha}\left(D^{\alpha}\left(D^{\alpha}\left(\ldots D^{\alpha}\left(\alpha_{0}\right)\right)\right)\right)}_{i}, \quad i=0,1,2, \ldots, n .
$$

Theorem 1. With Assumptions 1-3, virtual control laws (26), and feedback controller (25), the closed-loop system corresponding to (10) has the following properties:

(1) $y(t)$ asymptotically tracks the desired reference signal $y_{r}(t)$, i.e.,

$$
\begin{aligned}
\lim _{t \longrightarrow \infty}\left(y(t)-y_{r}(t)\right) & =0, \\
\text { when } \omega(t) & =0 .
\end{aligned}
$$

(2) $y(t)$ achieves the $H_{\infty}$ tracking performance, i.e.,

$$
\left\|\left(y(t)-y_{r}(t)\right)\right\|_{\alpha} \leq \gamma\|\omega(t)\|_{\alpha}
$$

for the zero initial condition when $\omega(t) \neq 0$, where $\gamma>0$.

Proof. The proof is given by constructing virtual control laws and a control law based on backstepping design approach.

Step 1: select the following Lyapunov function:

$$
V_{1}=\frac{1}{2} z_{1}^{2}
$$

Then, the fractional derivative of $V_{1}$ is given by

$$
\begin{aligned}
D^{\alpha} V_{1} & \leq z_{1} D^{\alpha} z_{1} \\
& =z_{1}\left[D^{\alpha} x_{1}-D^{\alpha} \alpha_{0}\right] \\
& =z_{1}\left(x_{2}+f_{1}+P_{1} \omega-D^{\alpha} \alpha_{0}\right) \\
& =z_{1}\left(z_{2}+\alpha_{1}+f_{1}+P_{1} \omega-D^{\alpha} \alpha_{0}\right) \\
& =z_{1} z_{2}+z_{1}\left(\alpha_{1}+f_{1}-D^{\alpha} \alpha_{0}\right)+z_{1} P_{1} \omega
\end{aligned}
$$

where the first inequality is generated from (3).

According to Lemma 4 , let $p=q=2$ and $\varepsilon=1 / \sqrt{2 \gamma}$; then, (34) can be estimated by

$$
\begin{aligned}
D^{\alpha} V_{1} & \leq z_{1} z_{2}+\gamma_{1} \omega^{T} \omega+z_{1}\left(\alpha_{1}+f_{1}-D^{\alpha} \alpha_{0}\right)+z_{1} \frac{P_{1} P_{1}^{T}}{4 \gamma_{1}} z_{1} \\
& =z_{1} z_{2}+\gamma_{1} \omega^{T} \omega+z_{1}\left(\alpha_{1}+f_{1}-D^{\alpha} \alpha_{0}+\frac{P_{1} P_{1}^{T}}{4 \gamma_{1}} z_{1}\right)
\end{aligned}
$$

Then, choose a virtual control law as

$$
\alpha_{1}=-k_{1} z_{1}-f_{1}+D^{\alpha} \alpha_{0}-\frac{P_{1} P_{1}^{T}}{4 \gamma_{1}} z_{1}
$$

where $k_{1}$ and $\gamma_{1}$ are positive constants and can be designed by us.

Furthermore, substituting (36) into (35) results in

$$
D^{\alpha} V_{1} \leq-k_{1} z_{1}^{2}+z_{1} z_{2}+\gamma_{1} \omega^{T} \omega
$$


Step 2:

Define a Lyapunov function as

$$
V_{2}=V_{1}+\frac{1}{2} z_{2}^{2}
$$

It follows from (28) and (27) that

$$
\begin{aligned}
D^{\alpha} z_{2} & =D^{\alpha} x_{2}-D^{\alpha} \alpha_{1} \\
& =x_{3}+f_{2}+P_{2} \omega-D^{\alpha} \alpha_{1},
\end{aligned}
$$

with

$$
\begin{aligned}
D^{\alpha} \alpha_{1}= & \frac{\partial \alpha_{1}}{\partial x_{1}}\left(x_{2}+f_{1}+P_{1} \omega\right)+\frac{\partial \alpha_{1}}{\partial \alpha_{0}} D^{\alpha} \alpha_{0} \\
& +\frac{\partial \alpha_{1}}{\partial D^{\alpha} \alpha_{0}} D^{\alpha}\left(D^{\alpha} \alpha_{0}\right) .
\end{aligned}
$$

Substituting (40) into (39) yields

$z_{2} D^{\alpha} z_{2}=z_{2}\left(x_{3}+f_{2}+\widehat{P}_{2} \omega-\frac{\partial \alpha_{1}}{\partial x_{1}}\left(x_{2}+f_{1}\right)-\sum_{s=0}^{1} \frac{\partial \alpha_{1}}{\partial \widehat{D}_{s}} \widehat{D}_{s+1}\right)$

Similar to (35), applying Lemma 4 to $\widehat{P}_{2} \omega,(41)$ can be estimated by

$$
\begin{aligned}
z_{2} D^{\alpha} z_{2} \leq & z_{2}\left(x_{3}+f_{2}-\frac{\partial \alpha_{1}}{\partial x_{1}}\left(x_{2}+f_{1}\right)-\sum_{s=0}^{1} \frac{\partial \alpha_{1}}{\partial \widehat{D}_{s}} \widehat{D}_{s+1}+\frac{\widehat{P}_{2} \widehat{P}_{2}^{T}}{4 \gamma_{2}} z_{2}\right) \\
& +\gamma_{2} \omega^{T} \omega .
\end{aligned}
$$

Then, the fractional derivative of $V_{2}$ is given by

$$
\begin{aligned}
D^{\alpha} V_{2} \leq & D^{\alpha} V_{1}+z_{2} D^{\alpha} z_{2} \\
\leq & -k_{1} z_{1}^{2}+\sum_{s=1}^{2} \gamma_{s} \omega^{T} \omega+z_{2} z_{3} \\
& +z_{2}\left[\alpha_{2}+z_{1}+f_{2}+\frac{\widehat{P}_{2} \widehat{P}_{2}^{T}}{4 \gamma_{2}} z_{2}-\frac{\partial \alpha_{1}}{\partial x_{1}}\left(x_{2}+f_{1}\right)\right. \\
& \left.-\sum_{s=0}^{1} \frac{\partial \alpha_{1}}{\partial \widehat{D}_{s}} \widehat{D}_{s+1}\right],
\end{aligned}
$$

where the first inequality is based on (37) and (3), respectively.
Similar to Step 1, the virtual control law is chosen as

$$
\begin{aligned}
\alpha_{2}= & -k_{2} z_{2}-z_{1}-f_{2}+\frac{\partial \alpha_{1}}{\partial x_{1}}\left(x_{2}+f_{1}\right)+\sum_{s=0}^{1} \frac{\partial \alpha_{1}}{\partial \widehat{D}_{s}} \widehat{D}_{s+1} \\
& -\frac{\widehat{P}_{2} \widehat{P}_{2}^{T}}{4 \gamma_{2}} z_{2}
\end{aligned}
$$

where $k_{2}$ is a positive constant. With virtual controller (43), (44) can be expressed as

$$
D^{\alpha} V_{2} \leq-k_{1} z_{1}^{2}-k_{2} z_{2}^{2}+z_{2} z_{3}+\sum_{s=1}^{2} \gamma_{s} \omega^{T} \omega
$$

Step $i(i=3, \ldots, n-1)$ :

In this step, we repeat the procedure in a recursive way. It follows from (27) and (28) that

$$
\begin{aligned}
D^{\alpha} z_{i} & =D^{\alpha} x_{i}-D^{\alpha} \alpha_{i-1} \\
& =x_{i+1}+f_{i}+P_{i} \omega-D^{\alpha} \alpha_{i-1},
\end{aligned}
$$

with

$$
D^{\alpha} \alpha_{i-1}=\sum_{s=1}^{i-1} \frac{\partial \alpha_{i-1}}{\partial x_{s}}\left(x_{s+1}+f_{s}+P_{s} \omega\right)+\sum_{s=0}^{i-1} \frac{\partial \alpha_{i-1}}{\partial \widehat{D}_{s}} \widehat{D}_{s+1}
$$

Substituting (47) into (46) yields

$$
\begin{aligned}
z_{i} D^{\alpha} z_{i}= & z_{i}\left(x_{i+1}+f_{i}+\widehat{P}_{i} \omega-\sum_{s=1}^{i-1} \frac{\partial \alpha_{i-1}}{\partial x_{s}}\left(x_{s+1}+f_{s}\right)\right. \\
& \left.-\sum_{s=0}^{i-1} \frac{\partial \alpha_{i-1}}{\partial \widehat{D}_{s}} \widehat{D}_{s+1}\right) \\
\leq & z_{i}\left(x_{i+1}+f_{i}-\sum_{s=1}^{i-1} \frac{\partial \alpha_{i-1}}{\partial x_{s}}\left(x_{s+1}+f_{s}\right)\right. \\
& \left.-\sum_{s=0}^{i-1} \frac{\partial \alpha_{i-1}}{\partial \widehat{D}_{s}} \widehat{D}_{s+1}+\frac{\widehat{P}_{i} \widehat{P}_{i}^{T}}{4 \gamma_{i}} z_{i}\right)+\gamma_{i} \omega^{T} \omega
\end{aligned}
$$

where the inequality is based on Lemma 4 with $p=$ $q=2$ and $\varepsilon=1 / \sqrt{2 \gamma}$.

With (48), it can be derived that the Lyapunov function

$$
V_{i}=V_{i-1}+\frac{1}{2} z_{i}^{2}
$$

has the following fractional derivative: 


$$
\begin{aligned}
D^{\alpha} V_{i} \leq & D^{\alpha} V_{i-1}+z_{i} D^{\alpha} z_{i} \\
\leq & -\sum_{s=1}^{i-1} k_{s} z_{s}^{2}+z_{i} z_{i+1}+\sum_{s=1}^{i} \gamma_{s} \omega^{T} \omega+z_{i} \\
& \cdot\left[\alpha_{i}+z_{i-1}+f_{i}-\sum_{s=1}^{i-1} \frac{\partial \alpha_{i-1}}{\partial x_{s}}\left(x_{s+1}+f_{s}\right)\right. \\
& \left.-\sum_{s=0}^{i-1} \frac{\partial \alpha_{i-1}}{\partial \widehat{D}_{s}} \widehat{D}_{s+1}+\frac{\widehat{P}_{i} \widehat{P}_{i}^{T}}{4 \gamma_{i}} z_{i}\right] .
\end{aligned}
$$

The virtual control law is chosen as

$$
\begin{aligned}
\alpha_{i}= & -k_{i} z_{i}-z_{i-1}-f_{i}+\sum_{s=1}^{i-1} \frac{\partial \alpha_{i-1}}{\partial x_{s}}\left(x_{s+1}+f_{s}\right) \\
& +\sum_{s=0}^{i-1} \frac{\partial \alpha_{i-1}}{\partial \widehat{D}_{s}} \widehat{D}_{s+1}-\frac{\widehat{P}_{i} \widehat{P}_{i}^{T}}{4 \gamma_{i}} z_{i},
\end{aligned}
$$

where $k_{i}$ is a positive constant. Next, substituting (51) into (50) results in

$$
D^{\alpha} V_{i} \leq-\sum_{s=1}^{i} k_{s} z_{s}^{2}+z_{i} z_{i+1}+\sum_{s=1}^{i} \gamma_{s} \omega^{T} \omega
$$

Step $n$ :

In the final step, select a Lyapunov function as

$$
V_{n}=V_{n-1}+\frac{1}{2} z_{n}^{2}
$$

It follows from (27) and (28) that

$$
\begin{aligned}
D^{\alpha} z_{n} & =D^{\alpha} x_{n}-D^{\alpha} \alpha_{n-1} \\
& =f+g u+f_{n}+P_{n} \omega-D^{\alpha} \alpha_{n-1},
\end{aligned}
$$

with

$D^{\alpha} \alpha_{n-1}=\sum_{s=1}^{n-1} \frac{\partial \alpha_{n-1}}{\partial x_{s}}\left(x_{s+1}+f_{s}+P_{s} \omega\right)+\sum_{s=0}^{n-1} \frac{\partial \alpha_{n-1}}{\partial \widehat{D}_{s}} \widehat{D}_{s+1}$

Substituting (55) into (54) yields

$$
\begin{aligned}
z_{n} D^{\alpha} z_{n}= & z_{n}\left(f+g u+f_{n}+\widehat{P}_{n} \omega-\sum_{s=1}^{n-1} \frac{\partial \alpha_{n-1}}{\partial x_{s}}\left(x_{s+1}+f_{s}\right)\right. \\
& \left.-\sum_{s=0}^{n-1} \frac{\partial \alpha_{n-1}}{\partial \widehat{D}_{s}} \widehat{D}_{s+1}\right) \\
\leq & z_{n}\left(f+g u+f_{n}-\sum_{s=1}^{n-1} \frac{\partial \alpha_{n-1}}{\partial x_{s}}\left(x_{s+1}+f_{s}\right)\right. \\
& \left.-\sum_{s=0}^{n-1} \frac{\partial \alpha_{n-1}}{\partial \widehat{D}_{s}} \widehat{D}_{s+1}+\frac{\widehat{P}_{n} \widehat{P}_{n}^{T}}{4 \gamma_{n}} z_{n}\right)+\gamma_{n} \omega^{T} \omega
\end{aligned}
$$

where the inequality is based on Lemma 4 with $p=$ $q=2$ and $\varepsilon=1 / \sqrt{2 \gamma}$.

Then, the fractional-order derivative of $V_{n}$ can be estimated by

$$
\begin{aligned}
D^{\alpha} V_{n} \leq & D^{\alpha} V_{n-1}+z_{n} D^{\alpha} z_{n} \\
\leq & -\sum_{s=1}^{n-1} k_{s} z_{s}^{2}+\sum_{s=1}^{n} \gamma_{s} \omega^{T} \omega+z_{n}\left[f+g u+f_{n}+z_{n-1}\right. \\
& \left.+\frac{\widehat{P}_{n} \widehat{P}_{n}^{T}}{4 \gamma_{n}} z_{n}-\sum_{s=1}^{n-1} \frac{\partial \alpha_{n-1}}{\partial x_{s}}\left(x_{s+1}+f_{s}\right)-\sum_{s=0}^{n-1} \frac{\partial \alpha_{n-1}}{\partial \widehat{D}_{s}} \widehat{D}_{s+1}\right] .
\end{aligned}
$$

Introduce the control law as follows:

$$
\begin{aligned}
u= & \frac{1}{g}\left[-f-\left(k_{n}+\frac{\widehat{P}_{n} \widehat{P}_{n}^{T}}{4 \gamma_{n}}\right) z_{n}-z_{n-1}-f_{n}\right. \\
& \left.+\sum_{s=1}^{n-1} \frac{\partial \alpha_{n-1}}{\partial x_{s}}\left(x_{s+1}+f_{s}\right)+\sum_{s=0}^{n-1} \frac{\partial \alpha_{n-1}}{\partial \widehat{D}_{s}} \widehat{D}_{s+1}\right],
\end{aligned}
$$

where $k_{i}$ is a positive constant. Next, substituting (58) into (57) results in

$$
D^{\alpha} V_{n} \leq-\sum_{s=1}^{n} k_{s} z_{s}^{2}+\sum_{s=1}^{n} \gamma_{s} \omega^{T} \omega
$$

(1) When $\omega(t)=0, D^{\alpha} V_{n} \leq 0$ can be guaranteed. Then, the output signal $y(t)$ asymptotically tracks the desired reference signal $y_{r}(t)$.

(2) When $\omega(t) \neq 0,(59)$ can be estimated by 

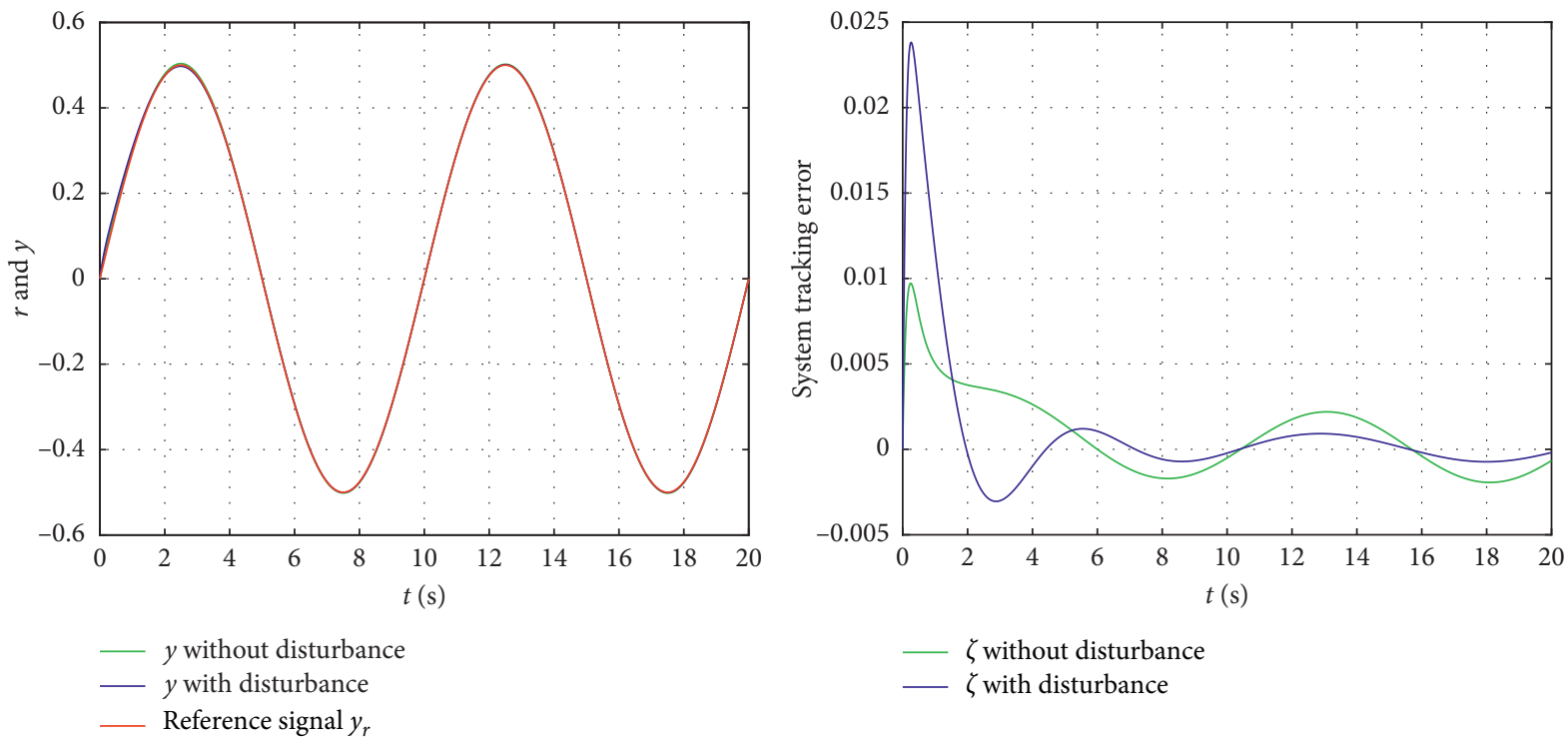

Figure 1: The tracking performance in Example 1.
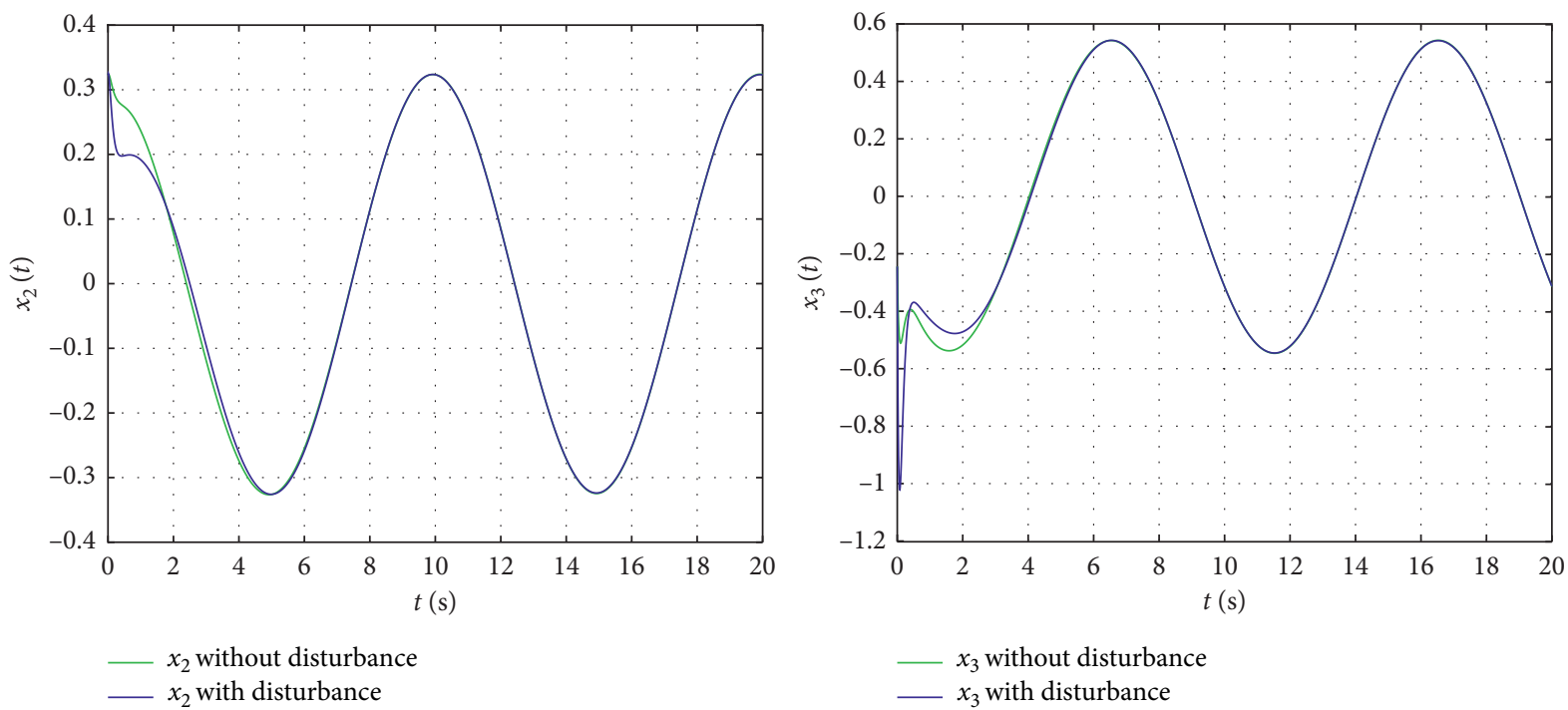

Figure 2: The state responses in Example 1.

$$
D^{\alpha} V_{n} \leq-k_{1} z_{1}^{2}+\bar{\gamma} \omega^{T} \omega,
$$

where $\bar{\gamma}=\sum_{s=1}^{n} \gamma_{s}$.

To simplify the notation, $V_{n}$ is abbreviated to be $V$. It follows from (53) that $V \geq 0$. For the $H_{\infty}$ performance, assume the initial condition is zero. Then, $V(0)=0$ is true. Calculate the Caputo fractional-order integral with order $\alpha$ of (60):

$$
V=D^{-\alpha}\left(D^{\alpha} V\right) \leq D^{-\alpha}\left(-k_{1} z_{1}^{T} z_{1}+\bar{\gamma} \omega^{T} \omega\right) .
$$

It means that

$$
0 \leq V \leq D^{-\alpha}\left(-k_{1} z_{1}^{T} z_{1}+\bar{\gamma} \omega^{T} \omega\right),
$$

i.e.,

$$
D^{-\alpha}\left(k_{1} z_{1}^{T} z_{1}\right) \leq D^{-\alpha}\left(\bar{\gamma} \omega^{T} \omega\right) .
$$

Then,

$$
\begin{aligned}
& \frac{k_{1}}{\Gamma(\alpha)} \int_{0}^{t} z_{1}^{T}(\sigma)(t-\sigma)^{\alpha-1} z_{1}(\sigma) \mathrm{d} \sigma \\
& \leq \frac{\bar{\gamma}}{\Gamma(\alpha)} \int_{0}^{t} \omega^{T}(\sigma)(t-\sigma)^{\alpha-1} \omega(\sigma) \mathrm{d} \sigma .
\end{aligned}
$$

Let $t \longrightarrow \infty$ : 


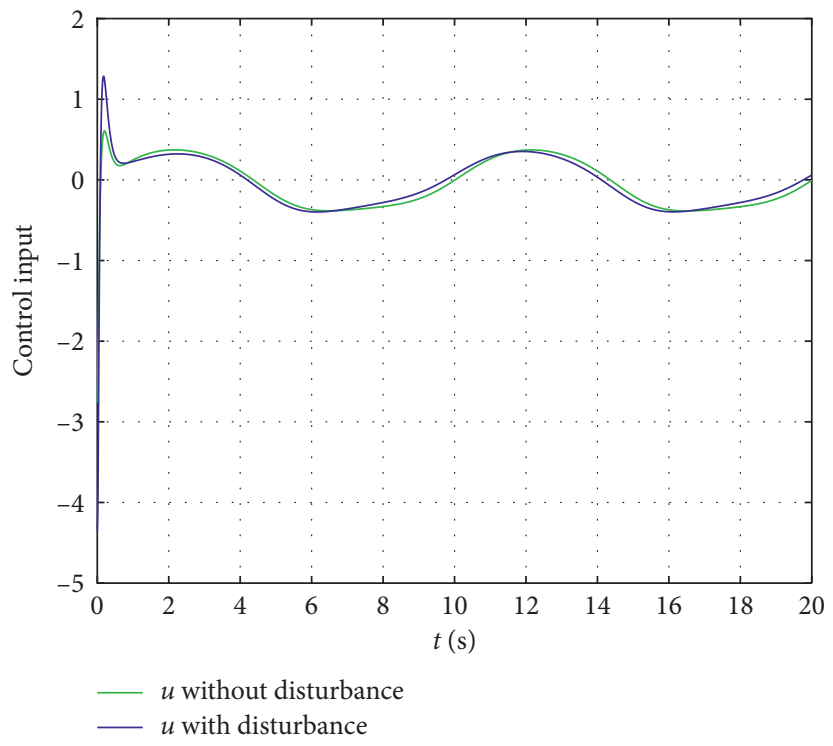

Figure 3: The control input in Example 1.

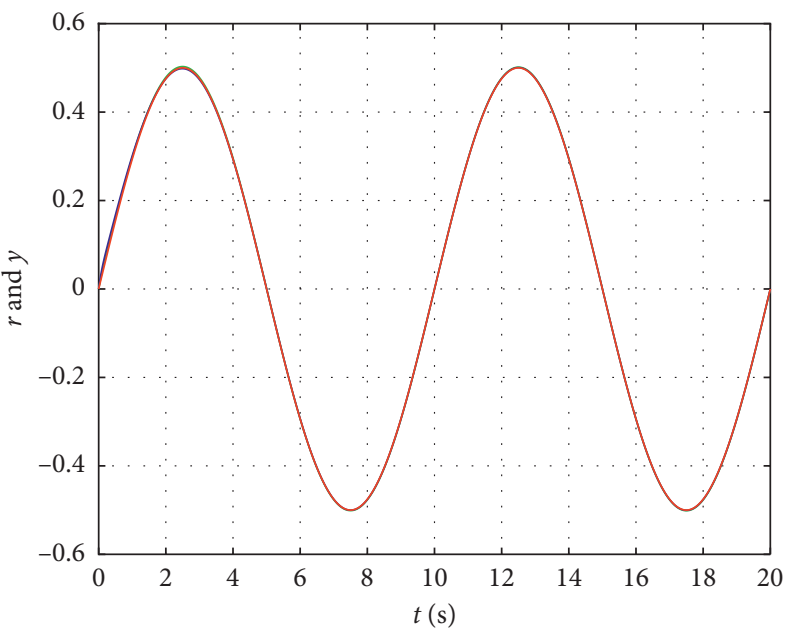

$y$ without disturbance $y$ with disturbance Reference signal $y_{r}$

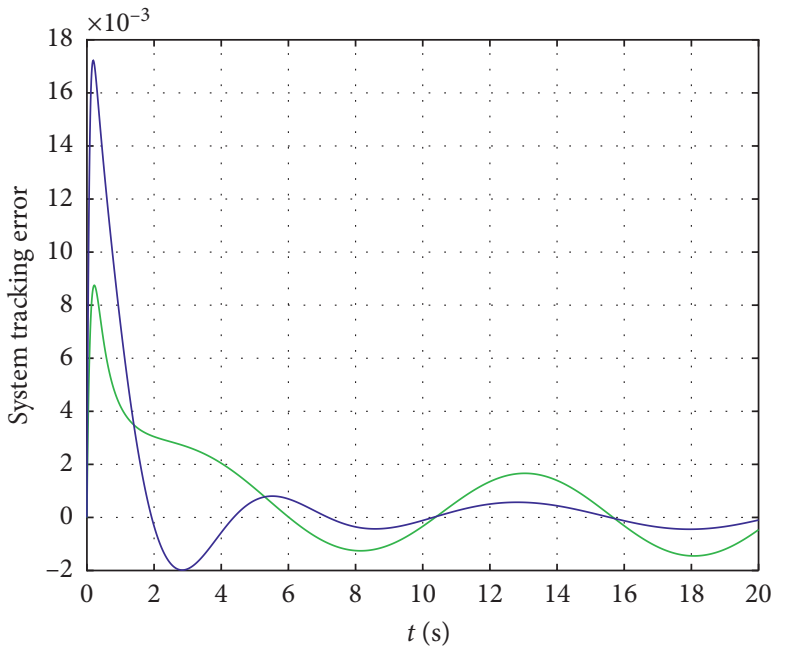

$\zeta$ without disturbance

$\zeta$ with disturbance

Figure 4: The tracking performance in Example 2.

$$
\left\|z_{1}(t)\right\|_{\alpha} \leq \sqrt{\frac{\bar{\gamma}}{k_{1}}}\|\omega(t)\|_{\alpha}
$$

i.e.,

$$
\left\|y(t)-y_{r}(t)\right\|_{\alpha} \leq \gamma\|\omega(t)\|_{\alpha},
$$

with $\gamma=\sqrt{\bar{\gamma} / k_{1}}$.

It means that the effect of disturbances on the output tracking error is attenuated to a given degree $\gamma$. Therefore, the $H_{\infty}$ tracking performance can be achieved.

\section{Simulation Results}

In order to verify the excellent properties of the proposed tracking controller, a numerical example is presented as Example 1, and two practical examples are given as Examples 2 and 3.

5.1. Example 1. Consider system (10) with $\alpha=0.93$, $f_{0}(z)=-z^{3}, f_{1}\left(\bar{x}_{1}\right)=0.1 x_{1}(t), f_{2}\left(\bar{x}_{2}\right)=0.5 x_{1}(t)+x_{2}(t)$, $f_{3}\left(\bar{x}_{3}\right)=0.2 x_{1}(t)-0.01 x_{2}(t)+x_{3}(t), \quad g(x)=x_{1}^{2}(t)$, $f(x)=x_{2}^{2}(t), P_{1}=P_{2}=P_{3}=0.1$, and $\omega(t)=\cos (t) e^{-0.5 t}$. The desired reference signal is set to $y_{r}(t)=0.5 \sin (\pi t / 5)$. The initial state $\left[x_{1}(0), x_{2}(0), x_{3}(0)\right]^{T}=[0,0.3226$, $-0.2443]^{T}$ and $z(0)=0.1$ are considered. 


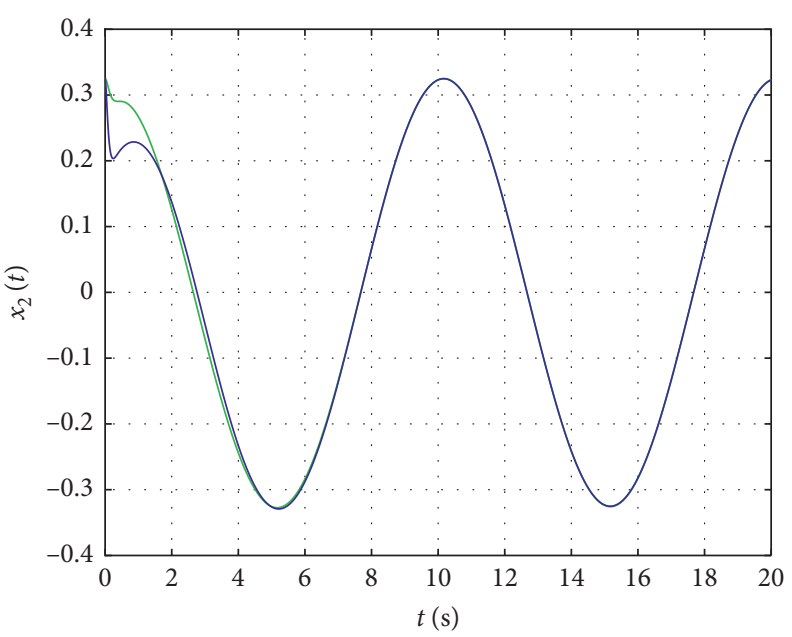

- $x_{2}$ without disturbance - $x_{2}$ with disturbance

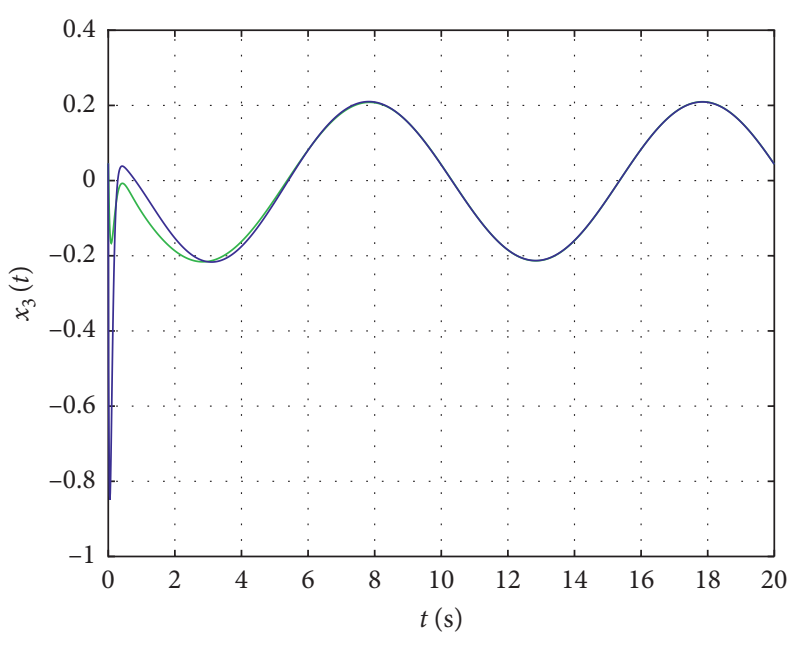

- $x_{3}$ without disturbance

$x_{3}$ with disturbance

Figure 5: The state responses in Example 2.

The virtual laws are chosen as

$$
\begin{aligned}
\alpha_{1}= & -\left(k_{1}+\frac{P_{1} P_{1}^{T}}{4 \gamma_{1}}\right) z_{1}-f_{1}+D^{\alpha} \alpha_{0} \\
\alpha_{2}= & -\left(k_{2}+\frac{\widehat{P}_{2} \widehat{P}_{2}^{T}}{4 \gamma_{2}}\right) z_{2}-z_{1}-f_{2}+\frac{\partial \alpha_{1}}{\partial x_{1}}\left(x_{2}+f_{1}\right) \\
& +\sum_{s=0}^{1} \frac{\partial \alpha_{1}}{\partial \widehat{D}_{s}} \widehat{D}_{s+1} .
\end{aligned}
$$

The adaptive control law is determined by

$$
\begin{aligned}
u= & \frac{1}{g}\left[-f-\left(k_{3}+\frac{\widehat{P}_{3} \widehat{P}_{3}^{T}}{4 \gamma_{3}}\right) z_{3}-z_{2}-f_{3}+\sum_{s=1}^{2} \frac{\partial \alpha_{2}}{\partial x_{s}}\left(x_{s+1}+f_{s}\right)\right. \\
& \left.+\sum_{s=0}^{2} \frac{\partial \alpha_{n-1}}{\partial \widehat{D}_{s}} \widehat{D}_{s+1}\right]
\end{aligned}
$$

where the design parameters are set to $k_{1}=12, k_{2}=10$, $k_{3}=10, \gamma_{1}=0.01, \gamma_{2}=0.2$, and $\gamma_{3}=4$.

Simulation results of Example 1 are shown as follows. Figure 1 gives the tracking performance, from which it can be concluded that the asymptotic tracking can be realized. It is obviously seen that the state variables are influenced by external disturbances in Figure 2. Figure 3 indicates the trajectories of the control input.

5.2. Example 2. Consider the fractional-order model of the magnetic leviation (FO-MAGLEV) suspension system in [49]:

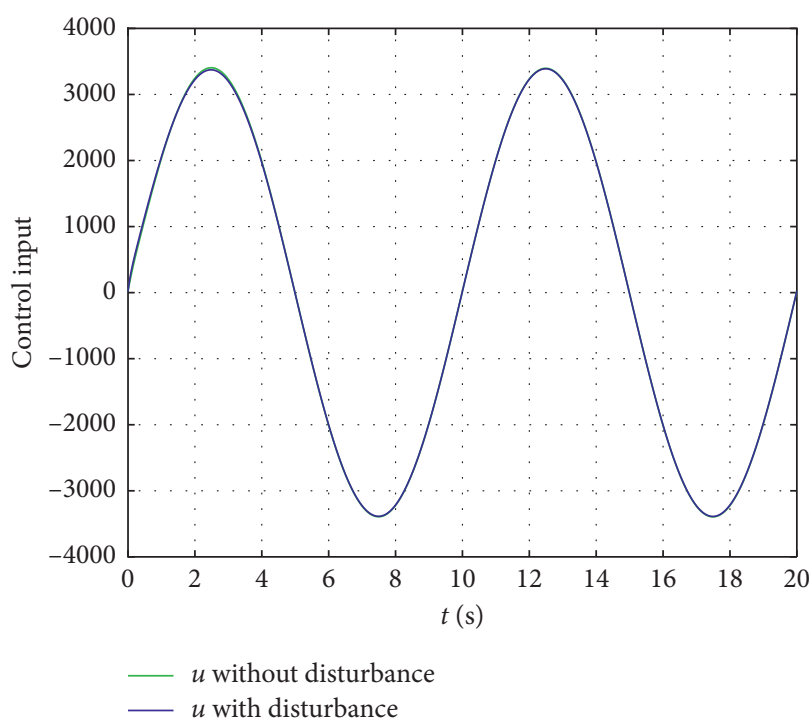

Figure 6: The control input in Example 2.

$$
\left\{\begin{array}{l}
D^{\alpha} x_{1}(t)=x_{2}(t)+P_{1} \omega(t), \\
D^{\alpha} x_{2}(t)=x_{3}(t)+P_{2} \omega(t), \\
D^{\alpha} x_{3}(t)=-6233.8 x_{1}(t)-65.2 x_{2}(t)-4.8 x_{3}(t) \\
\quad+0.9212 u+P_{3} \omega(t), \\
y(t)=x_{1}(t),
\end{array}\right.
$$

with $\quad \alpha=0.93, \quad P_{1}=0.1, \quad P_{2}=0, \quad P_{3}=-65.17 * 0.1$, $y_{r}(t)=0.5 \sin (\pi t / 5), \quad\left[x_{1}(0), x_{2}(0), x_{3}(0)\right]^{T}=[0,0.3226$, $0.046]^{T}$, and $\omega(t)=\cos (t) e^{-0.5 t}$.

The virtual laws and the adaptive control law are designed as (27) and (26) with the parameters chosen as 

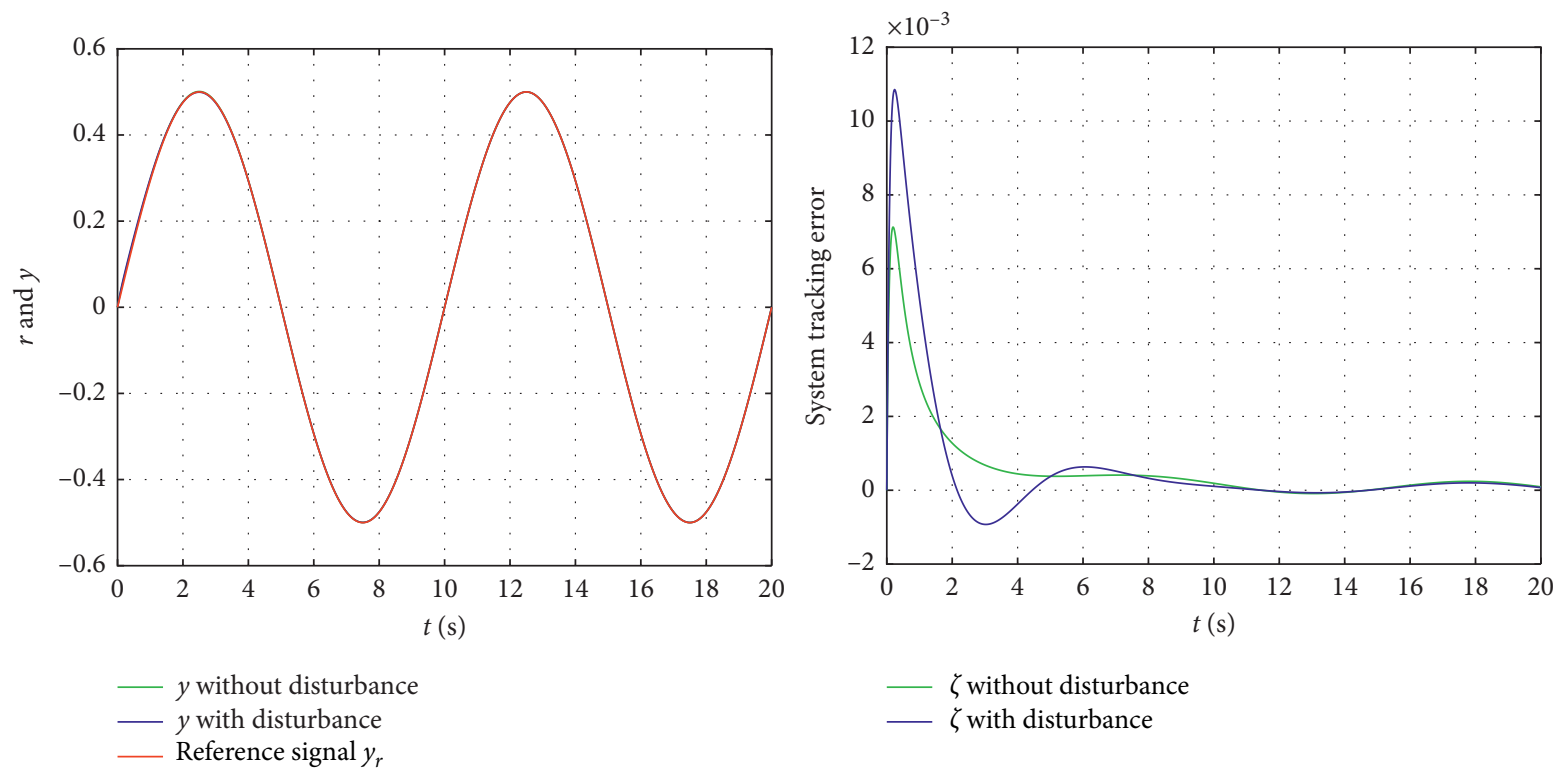

Figure 7: The tracking performance in Example 3.

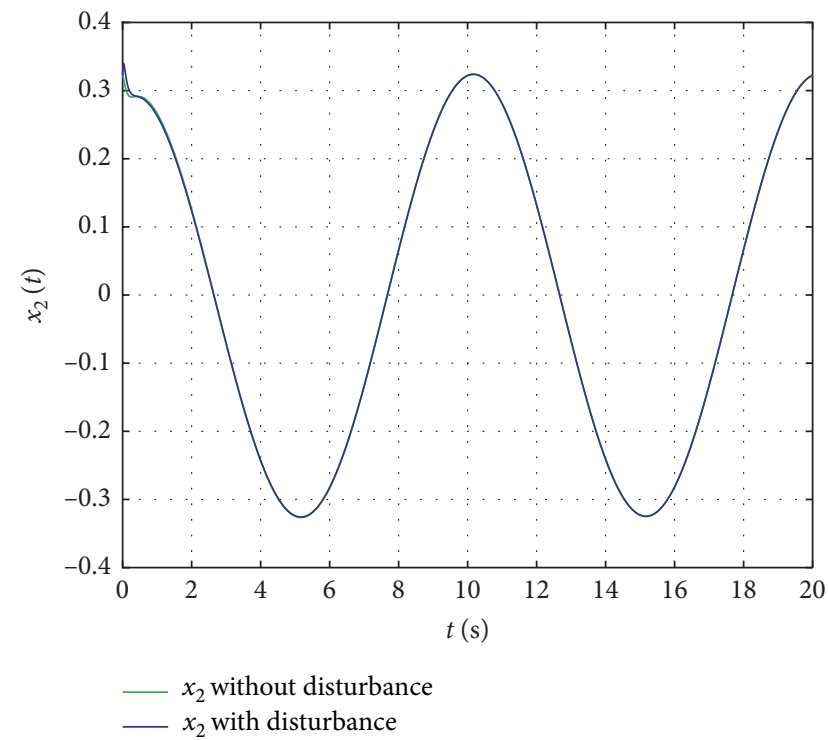

Figure 8: The state response in Example 3.

$k_{1}=16, k_{2}=12, k_{3}=10, \gamma_{1}=0.01, \gamma_{2}=0.1$, and $\gamma_{3}=5$. Figures 4-6 demonstrate the simulation results for Example 2. The tracking performance is illustrated in Figure 4. The state responses of Example 2 are shown in Figure 5. Figure 6 presents the control input of Example 2. It is seen that the tracking controller succeeds to make the output tracking error converge to zero without external disturbances and to attenuate the effect of disturbances on the tracking error.

5.3. Example 3. Consider the fractional-order model of the horizontal platform system (FO-HPS) given in [50]:

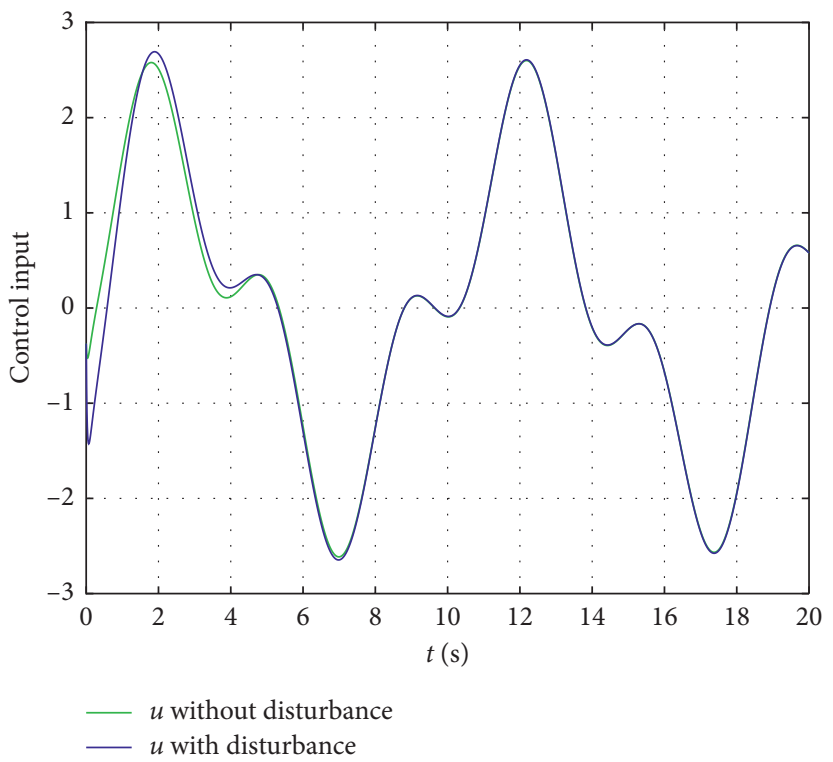

FIgURE 9: The control input in Example 3.

$$
\left\{\begin{array}{l}
D^{\alpha} x_{1}(t)=x_{2}(t)+P_{1} \omega(t), \\
D^{\alpha} x_{2}(t)=-\bar{a} x_{2}(t)-b \sin x_{1}(t)+l \sin x_{1}(t) \cos x_{1}(t) \\
\quad+h \cos \tau t+u(t)+P_{2} \omega(t), \\
y(t)=x_{1}(t)
\end{array}\right.
$$

with $\alpha=0.93, P_{1}=0, P_{2}=1, y_{r}(t)=0.5 \sin (\pi t / 5), \bar{a}=4 / 3$, $b=3.776, \quad l=4.6 * 10^{-6}, \quad h=3.4 / 4, \quad \tau=1.8$, $\left[x_{1}(0), x_{2}(0)\right]^{T}=[0,0.3226]^{T}$, and $\omega(t)=\cos (t) e^{-0.5 t}$. 
The virtual laws and the adaptive control law are determined by (27) and (26), where the design parameters are chosen as $k_{1}=10, k_{2}=10, \gamma_{1}=0.01$, and $\gamma_{2}=0.05$.

The simulation results of Example 3 are presented in Figures 7-9. The tracking performance of Example 3 is shown in Figure 7. The state responses of Example 3 are given in Figure 8. Figure 9 presents the control input of Example 3. It is observed that the reference signal can be tracked closely by the output signal and the impact of the external disturbances on the output tracking error can be reduced to a certain extent.

\section{Conclusion}

The ADD problem has been addressed for a type of nonlinear FOSs with time-varying disturbances and zero dynamics. With the help of the backstepping method, a stabilising state feedback control law has been constructed, which guarantees that asymptotic tracking is achieved without external disturbances and the effect of the external disturbances on the output tracking error can be reduced to a certain extent. In addition, a numerical example and two practical examples are given to validate the theoretical results.

\section{Data Availability}

The data used to support the findings of this study are available from the corresponding author upon request.

\section{Conflicts of Interest}

The authors declare that they have no conflicts of interest.

\section{Acknowledgments}

This work was supported in part by the Taishan Scholar Project of Shandong Province of China under Grant 2015162 and Grant tsqn201812093.

\section{References}

[1] I. Petráš, "A note on the fractional-order chuas system, chaos," Solitons \& Fractals, vol. 38, no. 1, pp. 140-147, 2008.

[2] N. Aguila-Camacho, M. A. Duarte-Mermoud, and J. A. Gallegos, "Lyapunov functions for fractional order systems," Communications in Nonlinear Science and Numerical Simulation, vol. 19, no. 9, pp. 2951-2957, 2014.

[3] I. Podlubny, Fractional Differential Equations: An Introductionto Fractional Derivatives, Fractional Differential Equations, to Methods of their Solution and Some of their Applications, vol. 198, Elsevier, Amsterdam, Netherlands, 1998.

[4] B. Xiao, H. K. Lam, G. Song, and H. Li, "Output-feedback tracking control for interval type-2 polynomial fuzzy-modelbased control systems," Neurocomputing, vol. 242, pp. 83-95, 2017.

[5] Y. Tang, X. Xing, H. R. Karimi, L. Kocarev, and J. Kurths, "Tracking control of networked multi-agent systems under new characterizations of impulses and its applications in robotic systems," IEEE Transactions on Industrial Electronics, vol. 63, no. 2, pp. 1299-1307, 2015.
[6] H. Wang, P. Shi, H. Li, and Q. Zhou, "Adaptive neural tracking control for a class of nonlinear systems with dynamic uncertainties," IEEE Transactions on Cybernetics, vol. 47, no. 10 , pp. 3075-3087, 2016.

[7] R. Sakthivel, S. Mohanapriya, C. K. Ahn, and H. Karimi, "Output tracking control for fractional-order positive switched systems with input time delay," IEEE Transactions on Circuits and Systems II: Express Briefs, vol. 66, no. 6, pp. 1013-1017, 2018.

[8] E. Ghotb Razmjou, S. K. Hosseini Sani, and J. Sadati, "Robust adaptive sliding mode control combination with iterative learning technique to output tracking of fractional-order systems," Transactions of the Institute of Measurement and Control, vol. 40, no. 6, pp. 1808-1818, 2018.

[9] C. Wang, M. Liang, and J. Gao, "Adaptive fuzzy output tracking control of a class of uncertain fractional order systems subject to unknown disturbance," IEEE Access, vol. 6, pp. 70655-70665, 2018.

[10] C. Hua, J. Ning, G. Zhao, and Y. Li, "Output feedback NN tracking control for fractional-order nonlinear systems with time-delay and input quantization," Neurocomputing, vol. 290, pp. 229-237, 2018.

[11] M. Krstić, I. Kanellakopoulos, and P. Kokotović, "Adaptive nonlinear control without over parametrization," Systems \& Control Letters, vol. 19, no. 3, pp. 177-185, 1992.

[12] J. Zhou and C. Wen, Adaptive Backstepping Control of Uncertain Systems: Nonsmooth Nonlinearities, Interactions or Time-Variations, Springer, Berlin, Germany, 2008.

[13] C. Liu, H. Wang, X. Liu, Y. Zhou, and S. Lu, "Adaptive prescribed performance tracking control for strict-feedback nonlinear systems with zero dynamics," International Journal of Robust and Nonlinear Control, vol. 29, no. 18, pp. 6507-6521, 2019.

[14] C. Liu, H. Wang, X. Liu, and Y. Zhou, "Adaptive finite-time fuzzy funnel control for non affine nonlinear systems," IEEE Transactions on Systems, Man, and Cybernetics: Systems, pp. 1-10, 2019, In press.

[15] C. Liu, X. Liu, H. Wang, Y. Zhou, S. Lu, and B. Xu, "Eventtriggered adaptive tracking control for uncertain nonlinear systems based on a new funnel function," ISA Transactions, 2019, In press.

[16] T. Shahiri, A. Ranjbar, R. Ghaderi, M. Karami, and S. Hosseinnia, "Adaptive backstepping chaos synchronization of fractional order coullet systems with mismatched parameters," https://arxiv.org/abs/1206.2026.

[17] D. Ding, D. Qi, Y. Meng, and L. Xu, "Adaptive mittag-leffler stabilization of commensurate fractional-order nonlinear systems," in Proceedings of the 53rd IEEE Conference on Decision and Control, pp. 6920-6926, IEEE, Los Angeles, CA, USA, December 2014.

[18] D. Ding, D. Qi, and Q. Wang, "Non-linear mittag-leffler stabilisation of commensurate fractional-order non-linear systems," IET Control Theory \& Applications, vol. 9, no. 5, pp. 681-690, 2015.

[19] D. Ding, D. Qi, J. Peng, and Q. Wang, “Asymptotic pseudostate stabilization of commensurate fractional-order nonlinear systems with additive disturbance," Nonlinear Dynamics, vol. 81, no. 1-2, pp. 667-677, 2015.

[20] Y. Wei, Y. Chen, S. Liang, and Y. Wang, "A novel algorithm on adaptive backstepping control of fractional order systems," Neurocomputing, vol. 165, pp. 395-402, 2015.

[21] Y. Wei, W. T. Peter, Z. Yao, and Y. Wang, "Adaptive backstepping output feedback control for a class of nonlinear fractional order systems," Nonlinear Dynamics, vol. 86, no. 2, pp. 1047-1056, 2016. 
[22] J. C. Willems and C. Commault, "Disturbance decoupling by measurement feedback with stability or pole placement," SIAM Journal on Control and Optimization, vol. 19, no. 4, pp. 490-504, 1981.

[23] G. Basile and G. Marro, Controlled and Conditioned Invariants in Linear System Theory, Prentice-Hall, Englewood Cliffs, NJ, USA, 1992.

[24] H. Nijmeijer and A. Van der Schaft, Nonlinear Dynamical Control Systems, vol. 175, Springer, Berlin, Germany, 1990.

[25] S. Weiland and J. C. Willems, "Almost disturbance decoupling with internal stability," IEEE Transactions on Automatic Control, vol. 34, no. 3, pp. 277-286, 1989.

[26] R. Marino, W. Respondek, and A. Van der Schaft, "Almost disturbance decoupling for single-input single-output nonlinear systems," IEEE Transactions on Automatic Control, vol. 34, no. 9, pp. 1013-1017, 1989.

[27] X. Liu, A. Jutan, and S. Rohani, "Almost disturbance decoupling of mimo nonlinear systems and application to chemical processes," Automatica, vol. 40, no. 3, pp. 465-471, 2004.

[28] Z. Lin, "Almost disturbance decoupling with global asymptotic stability for nonlinear systems with disturbance-affected unstable zero dynamics," Systems \& Control Letters, vol. 33, no. 3, pp. 163-169, 1998.

[29] Z. Lin, X. Bao, and B. M. Chen, "Further results on almost disturbance decoupling with global asymptotic stability for nonlinear systems," Automatica, vol. 35, no. 4, pp. 709-717, 1999.

[30] R. Marino and P. Tomei, "Nonlinear output feedback tracking with almost disturbance decoupling," IEEE Transactions on Automatic Control, vol. 44, no. 1, pp. 18-28, 1999.

[31] C. Qian and W. Lin, "Almost disturbance decoupling for a class of high-order nonlinear systems," IEEE Transactions on Automatic Control, vol. 45, no. 6, pp. 1208-1214, 2000.

[32] C.-C. Chen, T.-L. Chien, and C.-L. Wei, "Tracking and almost disturbance decoupling for nonlinear systems with uncertainties," JSME International Journal Series C Mechanical Systems, Machine Elements and Manufacturing, vol. 46, no. 3, pp. 1100-1107, 2003.

[33] B. Chen, S. Tong, and X. Liu, "Fuzzy approximate disturbance decoupling of mimo nonlinear systems by backstepping approach," Fuzzy Sets and Systems, vol. 158, no. 10, pp. 1097-1125, 2007.

[34] C.-C. Chen, Y.-F. Lin, and M.-H. Chen, "Nonlinear tracking with almost disturbance decoupling and its application to ball and beam system," Journal of the Chinese Institute of Engineers, vol. 30, no. 3, pp. 545-551, 2007.

[35] H. Chu, C. Qian, J. Yang, S. Xu, and Y. Liu, "Almost disturbance decoupling for a class of nonlinear systems via sampled-data output feedback control," International Journal of Robust and Nonlinear Control, vol. 26, no. 10, pp. 22012215, 2016.

[36] Y.-M. Fu, A.-G. Wu, and G.-R. Duan, "Almost disturbance decoupling for a class of inherently nonlinear systems," International Journal of Control, Automation and Systems, vol. 7, no. 2, pp. 325-330, 2009.

[37] Z. Zhang, X. Liu, Y. Liu, C. Lin, and B. Chen, "Fixed-time almost disturbance decoupling of nonlinear time-varying systems with multiple disturbances and dead-zone input," Information Sciences, vol. 450, pp. 267-283, 2018.

[38] X. Liu, Y. Liu, Y. Zhou, and Y. Jia, "The finite-time almost disturbance decoupling for nonlinear systems," International Journal of Systems Science, vol. 49, no. 10, pp. 2243-2256, 2018.
[39] B.-Z. Guo and Z.-H. Wu, "Output tracking for a class of nonlinear systems with mismatched uncertainties by active disturbance rejection control," Systems \& Control Letters, vol. 100, pp. 21-31, 2017.

[40] Z.-H. Wu and B.-Z. Guo, "Approximate decoupling and output tracking for MIMO nonlinear systems with mismatched uncertainties via ADRC approach," Journal of the Franklin Institute, vol. 355, no. 9, pp. 3873-3894, 2018.

[41] H.-C. Zhou, C. Lv, B.-Z. Guo, and Y. Chen, "Mittag-leffler stabilization for an unstable time-fractional anomalous diffusion equation with boundary control matched disturbance," International Journal of Robust and Nonlinear Control, vol. 29, no. 13, pp. 4384-4401, 2019.

[42] H. Zhou, "Output-based disturbance rejection control for 1-d anti-stable Schrödinger equation with boundary input matched unknown disturbance," International Journal of Robust and Nonlinear Control, vol. 27, no. 18, pp. 4686-4705, 2017.

[43] Z. Zhao, C. K. Ahn, and H.-X. Li, "Boundary anti-disturbance control of a spatially nonlinear flexible string system," IEEE Transactions on Industrial Electronics, vol. 67, no. 6, pp. 4846-4856, 2020.

[44] Z. Zhao, X. He, and C. K. Ahn, "Boundary disturbance observer-based control of a vibrating single-link flexible manipulator," IEEE Transactions on Systems, Man, and Cybernetics: Systems, pp. 1-9, 2019, In press.

[45] M. P. Aghababa, "Comments on "adaptive fuzzy H tracking design of SISO uncertain nonlinear fractional order timedelay systems" [nonlinear dyn. 69 (2012) 1639-1650]," Nonlinear Dynamics, vol. 70, no. 4, pp. 2511-2513, 2012.

[46] T.-C. Lin, C.-H. Kuo, T.-Y. Lee, and V. E. Balas, "Adaptive fuzzy $\mathrm{H}$ tracking design of SISO uncertain nonlinear fractional order time-delay systems," Nonlinear Dynamics, vol. 69, no. 4, pp. 1639-1650, 2012.

[47] A. A. A. Kilbas, H. M. Srivastava, and J. J. Trujillo, Theory and Applications of Fractional Differential Equations, vol. 204, Elsevier Science Limited, Berlin, Germany, 2006.

[48] Y. Li, Y. Chen, and I. Podlubny, "Mittag-leffler stability of fractional order nonlinear dynamic systems," Automatica, vol. 45, no. 8, pp. 1965-1969, 2009.

[49] S. Pashaei and M. Badamchizadeh, "A new fractional-order sliding mode controller via a nonlinear disturbance observer for a class of dynamical systems with mismatched disturbances," ISA Transactions, vol. 63, pp. 39-48, 2016.

[50] T. Binazadeh, "Finite-time tracker design for uncertain nonlinear fractional-order systems," Journal of Computational and Nonlinear Dynamics, vol. 11, no. 4, Article ID 041028, 2016. 